\title{
Screen Transversal Lightlike Submanifolds of Indefinite Cosymplectic Manifolds
}

\author{
RAm Shankar Gupta (*) - A. Sharfuddin (**)
}

ABSTRACT - In this paper, we introduce screen transversal lightlike submanifolds of indefinite Cosymplectic manifolds. Then, we study the geometry of this new class and its subspaces with example.

\section{Introduction.}

In the theory of submanifolds of semi-Riemannian manifolds it is interesting to study the geometry of lightlike submanifolds due to the fact that the intersection of normal vector bundle and the tangent bundle is non-trivial making it more interesting and remarkably different from the study of non-degenerate submanifolds.The geometry of lightlike submanifolds of indefinite Kaehler manifolds was studied by Duggal and Bejancu [2] and a general notion of screen transversal lightlike submanifolds of indefinite Kaehler manifolds was introduced by Sahin [3]. However, a general notion of screen transversal lightlike submanifolds of indefinite Cosymplectic manifolds has not been introduced as yet.

In section 1, we have collected the formulae and information which are useful in subsequent sections. In section 2, we define screen transversal, screen transversal anti-invariant and radical transversal lightlike submanifolds. In section 3, we obtained a characterization of screen transversal anti-invariant lightlike submanifolds as well as a condition for in-

(*) Indirizzo dell'A.: University School of Basic and Applied Sciences, Guru Gobind Singh Indraprastha University, Kashmere Gate, Delhi-110006, India.

Email: ramshankar.gupta@gmail.com

(**) Indirizzo dell'A.: Department of Mathematics, Faculty of Natural Sciences, Jamia Millia Islamia (Central University), New Delhi-11025, India.

E-mail: sharfuddin_ahmad12@yahoo.com

AMS2000 MSC Codes: 53C15, 53C40, 53C50, 53D15. 
duced connection to be a metric connection and provided an example of STanti-invariant lightlike submanifold of $R_{2}^{9}$. In section 4 , we have studied radical screen transversal lightlike submanifolds.

\section{Preliminaries.}

An odd-dimensional semi-Riemannian manifold $\bar{M}$ is said to be an indefinite almost contact metric manifold if there exist structure tensors $\{\phi, V, \eta, \bar{g}\}$, where $\phi$ is a $(1,1)$ tensor field, $V$ a vector field, $\eta$ a 1 -form and $\bar{g}$ is the semi-Riemannian metric on $\bar{M}$ satisfying

$$
\left\{\begin{array}{l}
\phi^{2} X=-X+\eta(X) V, \quad \eta \circ \phi=0, \quad \phi V=0, \quad \eta(V)=\varepsilon \\
\bar{g}(\phi X, \phi Y)=\bar{g}(X, Y)-\varepsilon \eta(X) \eta(Y), \quad \bar{g}(X, V)=\eta(X)
\end{array}\right.
$$

for any $X, Y \in T \bar{M}$, where $T \bar{M}$ denotes the Lie algebra of vector fields on $\bar{M}$ and $\varepsilon= \pm 1$.

An indefinite almost contact metric manifold $\bar{M}$ is called an indefinite Cosymplectic manifold if $[1,4]$,

$$
\left(\bar{\nabla}_{X} \phi\right) Y=0 \quad \text { and } \quad \bar{\nabla}_{X} V=0
$$

for any $X, Y \in T \bar{M}$, where $\bar{\nabla}$ denote the Levi-Civita connection on $\bar{M}$.

A submanifold $M^{m}$ immersed in a semi-Riemannian manifold $\left\{\bar{M}^{m+k}, \bar{g}\right\}$ is called a lightlike submanifold if it admits a degenerate metric $g$ induced from $\bar{g}$ whose radical distribution $\operatorname{Rad}(T M)$ is of rank $r$, where $1 \leq r \leq m$.

Now, $\operatorname{Rad}(T M)=T M \cap T M^{\perp}$, where

$$
T M^{\perp}=\bigcup_{x \in M}\left\{u \in T_{x} \bar{M}: \bar{g}(u, v)=0, \forall v \in T_{x} M\right\} .
$$

Let $S(T M)$ be a screen distribution which is a semi-Riemannian complementary distribution of $\operatorname{Rad}(T M)$ in $T M$, that is, $T M=\operatorname{Rad}(T M) \perp S(T M)$.

Also, there exists a screen transversal vector bundle $S\left(T M^{\perp}\right)$, which is a semi-Riemannian complementary vector bundle of $\operatorname{Rad}(T M)$ in $T M^{\perp}$. Since, for any local basis $\left\{\xi_{i}\right\}$ of $\operatorname{Rad}(T M)$, there exists a local frame $\left\{N_{i}\right\}$ of sections with values in the orthogonal complement of $S\left(T M^{\perp}\right)$ in $[S(T M)]^{\perp}$ such that $\bar{g}\left(\xi_{i}, N_{j}\right)=\delta_{i j}$ and $\bar{g}\left(N_{i}, N_{j}\right)=0$, it follows that there exists a lightlike transversal vector bundle ltr(TM) locally spanned by $\left\{N_{i}\right\}(c f$. [2], page 144). Let $\operatorname{tr}(T M)$ be the complementary 
(but not orthogonal) vector bundle to $T M$ in $\left.T \bar{M}\right|_{M}$. Then

$$
\left\{\begin{array}{l}
\operatorname{tr}(T M)=l \operatorname{tr}(T M) \perp S\left(T M^{\perp}\right) \\
\left.T \bar{M}\right|_{M}=S(T M) \perp[\operatorname{Rad}(T M) \oplus \operatorname{ltr}(T M)] \perp S\left(T M^{\perp}\right) .
\end{array}\right.
$$

A submanifold $\left(M, g, S(T M), S\left(T M^{\perp}\right)\right)$ of $\bar{M}$ is said to be

(i) -lightlike if $\quad r<\min \{m, k\}$;

(ii) Coisotropic if $\quad r=k<m, \quad S\left(T M^{\perp}\right)=\{0\}$;

(iii) Isotropic if $\quad r=m<k, \quad S(T M)=\{0\}$;

(iv) Totally lightlike if $\quad r=m=k, \quad S(T M)=\{0\}=S\left(T M^{\perp}\right)$.

Let $\bar{\nabla}, \nabla$ and $\nabla^{t}$ denote the linear connections on $\bar{M}, M$ and vector bundle $\operatorname{tr}(T M)$, respectively. Then the Gauss and Weingarten formulae are given by

$$
\begin{aligned}
& \bar{\nabla}_{X} Y=\nabla_{X} Y+h(X, Y), \quad \forall X, Y \in \Gamma(T M), \\
& \bar{\nabla}_{X} U=-A_{U} X+\nabla_{X}^{t} U, \quad \forall U \in \Gamma(\operatorname{tr}(T M)),
\end{aligned}
$$

where $\left\{\nabla_{X} Y, A_{U} X\right\}$ and $\left\{h(X, Y), \nabla_{X}^{t} U\right\}$ belong to $\Gamma(T M)$ and $\Gamma(\operatorname{tr}(T M))$, respectively and $A_{U}$ is the shape operator of $M$ with respect to $U$. Moreover, according to the decomposition (1.4), $h^{l}, h^{s}$ are $\Gamma(\operatorname{ltr}(T M))$-valued and $\Gamma\left(S\left(T M^{\perp}\right)\right)$-valued lightlike second fundamental form and screen second fundamental form of $M$, respectively, then

$$
\begin{gathered}
\bar{\nabla}_{X} Y=\nabla_{X} Y+h^{l}(X, Y)+h^{s}(X, Y), \quad \forall X, Y \in \Gamma(T M), \\
\bar{\nabla}_{X} N=-A_{N} X+\nabla_{X}^{l}(N)+D^{s}(X, N), \quad N \in \Gamma(\operatorname{ltr}(T M)), \\
\bar{\nabla}_{X} W=-A_{W} X+\nabla_{X}^{s}(W)+D^{l}(X, W), \quad W \in \Gamma\left(S\left(T M^{\perp}\right)\right),
\end{gathered}
$$

where $D^{l}(X, W), D^{s}(X, N)$ are the projections of $\nabla^{t}$ on $\Gamma(\operatorname{ltr}(T M))$ and $\Gamma\left(S\left(T M^{\perp}\right)\right)$, respectively and $\nabla^{l}, \nabla^{s}$ are linear connections on $\Gamma(\operatorname{ltr}(T M))$ and $\Gamma\left(S\left(T M^{\perp}\right)\right.$ ), respectively. We call $\nabla^{l}, \nabla^{s}$ the lightlike and screen transversal connections on $M$, and $A_{N}, A_{W}$ are shape operators on $M$ with respect to $N$ and $W$, respectively. Using (1.5) and (1.7)-(1.9), we obtain

$$
\begin{gathered}
\bar{g}\left(h^{s}(X, Y), W\right)+\bar{g}\left(Y, D^{l}(X, W)\right)=g\left(A_{W} X, Y\right), \\
\bar{g}\left(D^{s}(X, N), W\right)=\bar{g}\left(N, A_{W} X\right) .
\end{gathered}
$$

Let $\bar{P}$ denote the projection of $T M$ on $S(T M)$ and let $\nabla^{*}, \nabla^{* t}$ denote the linear connections on $S(T M)$ and $\operatorname{Rad}(T M)$, respectively. Then from the 
decomposition of tangent bundle of lightlike submanifold, we have

$$
\begin{gathered}
\nabla_{X} \bar{P} Y=\nabla_{X}^{*} \bar{P} Y+h^{*}(X, \bar{P} Y), \\
\nabla_{X} \xi=-A_{\xi}^{*} X+\nabla_{X}^{* t} \xi,
\end{gathered}
$$

for $X, Y \in \Gamma(T M)$ and $\xi \in \Gamma(\operatorname{RadTM})$, where $h^{*}, A^{*}$ are the second fundamental form and shape operator of distributions $S(T M)$ and $\operatorname{Rad}(T M)$, respectively.

From (1.12) and (1.13), we get

$$
\begin{gathered}
\bar{g}\left(h^{l}(X, \bar{P} Y), \xi\right)=g\left(A_{\xi}^{*} X, \bar{P} Y\right), \\
\bar{g}\left(h^{*}(X, \bar{P} Y), N\right)=g\left(A_{N} X, \bar{P} Y\right), \\
\bar{g}\left(h^{l}(X, \xi), \xi\right)=0, \quad A_{\xi}^{*} \xi=0 .
\end{gathered}
$$

In general, the induced connection $\nabla$ on $M$ is not a metric connection. Since $\bar{\nabla}$ is a metric connection, from (1.7), we obtain

$$
\left(\nabla_{X} g\right)(Y, Z)=\bar{g}\left(h^{l}(X, Y), Z\right)+\bar{g}\left(h^{l}(X, Z), Y\right) .
$$

However, it is important to note that $\nabla^{*}, \nabla^{* t}$ are metric connections on $S(T M)$ and $\operatorname{Rad}(T M)$, respectively.

\section{Screen transversal lightlike submanifolds.}

In this section, we introduce screen transversal $(S T)$, radical screen transversal and screen transversal anti-invariant lightlike submanifolds of indefinite Cosymplectic manifolds.

LEMma 2.1. Let $M$ be an r-lightlike submanifold of an indefinite Cosymplectic manifold $\bar{M}$. Suppose that $\phi R a d T M$ is a vector subbundle of $S\left(T M^{\perp}\right)$. Then, $\phi$ ttrTM is also vector subbundle of the screen transversal bundle $S\left(T M^{\perp}\right)$. Moreover, $\phi$ ltrTM $\cap \phi R a d T M=\{0\}$.

Proof. Let us assume that ltrTM is invariant with respect to $\phi$. Then by the definition of a lightlike submanifold, there exist vector fields $\xi \in \Gamma(\operatorname{RadTM})$ and $N \in \Gamma(\operatorname{ltr}(T M))$ such that $\bar{g}(\xi, N)=1$. Also from (1.1), we get

$$
\bar{g}(\phi \xi, \phi N)=\bar{g}(\xi, N)-\varepsilon \eta(N) \eta(\xi)=\bar{g}(\xi, N)=1 .
$$

However, if $\phi N \in \Gamma(\operatorname{ltr}(T M))$ then by the hypothesis we get $\bar{g}(\phi \xi, \phi N)=0$. 
Hence, we obtain a contradiction which implies that $\phi N$ does not belong to $\operatorname{ltr}(T M)$.

Now, suppose that $\phi N \in \Gamma(S(T M))$. Then, in a similar way, we have

$$
\bar{g}(\phi \xi, \phi N)=\bar{g}(\xi, N)-\varepsilon \eta(N) \eta(\xi)=\bar{g}(\xi, N)=1
$$

which is again a contradiction. Thus $\phi N$ does not belong to $S(T M)$.

We can also obtain that $\phi N$ does not belong to RadTM. Then, from the decomposition of a lightlike submanifold, we conclude that $\phi N \in S\left(T M^{\perp}\right)$.

Now, suppose that there exists a vector field $X \in \Gamma(\phi l t r T M \cap \phi R a d T M)$. Then, we have $\bar{g}(X, \phi N)=0$, since $X \in \Gamma(\phi l t r T M)$. However, for an $r$ lightlike submanifold there exists some vector fields $\phi X \in \Gamma(\operatorname{RadTM})$ for $X \in \Gamma \phi(\operatorname{RadTM})$ such that $\bar{g}(\phi X, N) \neq 0$. Since $\phi$ is skew symmetric, we get

$$
0 \neq \bar{g}(\phi X, N)=-\bar{g}(X, \phi N)=0
$$

which is a contradiction. Thus, proof is complete.

DEFINITION 2.1. Let $M$ be an r-lightlike submanifold of an indefinite Cosymplectic manifold $\bar{M}$. Then $M$ is called screen transversal lightlike (ST-lightlike) submanifold of $\bar{M}$ if there exist a screen transversal vector bundle $S\left(T M^{\perp}\right)$ such that $\phi R a d T M \subset S\left(T M^{\perp}\right)$.

DEFINITION 2.2. Let $M$ be ST-lightlike submanifold of an indefinite Cosymplectic manifold $\bar{M}$. Then

(a) $M$ is a radical ST-lightlike submanifold of $\bar{M}$ if $S(T M)$ is invariant with respect to $\phi$.

(b) $M$ is a $S T$-anti-invariant lightlike submanifold of $\bar{M}$ if $S(T M)$ is screen transversal with respect to $\phi$ i.e. $\phi S(T M) \subset S\left(T M^{\perp}\right)$.

From Lemma 2.1 and Definition 2.1, it follows that $\phi l t r T M \subset S\left(T M^{\perp}\right)$. Also it is obvious that there are no co-isotropic and totally lightlike $S T$ lightlike submanifolds of indefinite Cosymplectic manifolds. It is important to point out that $\phi R a d T M$ and $\phi l t r T M$ are not orthogonal otherwise $S\left(T M^{\perp}\right)$ would be degenerate.

For $S T$-anti-invariant lightlike submanifold $M$ of an indefinite Cosymplectic manifold $\bar{M}$ with structure vector field tangent to $M$, we have

$$
S\left(T M^{\perp}\right)=\phi(\operatorname{RadTM}) \oplus \phi(\operatorname{ltr} T M) \perp \phi\left(D^{\prime}\right) \perp D_{0}
$$

where $S(T M)=D^{\prime} \perp\{V\}$ and $D_{0}$ is complementary distribution orthogonal to $\phi(\operatorname{RadTM}) \oplus \phi(l t r T M) \perp \phi\left(D^{\prime}\right)$ in $S\left(T M^{\perp}\right)$. 
Proposition 2.1. Let M be ST-lightlike submanifold of an indefinite Cosymplectic manifold $\bar{M}$. Then the distribution $D_{0}$ is invariant with respect to $\phi$.

Proof. For $X \in \Gamma\left(D_{0}\right), \xi \in \Gamma(\operatorname{RadTM}), N \in \Gamma(\operatorname{ltrTM})$, we have

$$
\bar{g}(\phi X, \xi)=-\bar{g}(X, \phi \bar{\xi})=0 \quad \text { and } \quad \bar{g}(\phi X, N)=-\bar{g}(X, \phi N)=0
$$

which implies that $\phi\left(D_{0}\right) \cap R a d T M=\{0\}$ and $\phi\left(D_{0}\right) \cap \operatorname{ltr}(T M)=\{0\}$. From (1.1), we get

$$
\bar{g}(\phi X, \phi \xi)=\bar{g}(X, \xi)-\varepsilon \eta(X) \eta(\xi)=\bar{g}(X, \xi)=0
$$

and

$$
\bar{g}(\phi X, \phi N)=\bar{g}(X, N)-\varepsilon \eta(X) \eta(N)=\bar{g}(X, N)=0
$$

which shows that $\phi\left(D_{0}\right) \cap \phi(R a d T M)=\{0\}$ and $\phi\left(D_{0}\right) \cap \phi(\operatorname{ltr}(T M))=\{0\}$. Moreover, since $\phi(S(T M))$ and $D_{0}$ are orthogonal, we obtain

$$
\bar{g}(\phi X, Z)=-\bar{g}(X, \phi Z)=0
$$

and

$$
\bar{g}(\phi X, \phi Z)=\bar{g}(X, Z)-\varepsilon \eta(X) \eta(Z)=\bar{g}(X, Z)=0
$$

for $Z \in \Gamma(S(T M)), \phi Z \in \Gamma(\phi(S(T M)))$, which shows that

$$
\phi\left(D_{0}\right) \cap S(T M)=\{0\} \quad \text { and } \quad \phi\left(D_{0}\right) \cap \phi(S(T M))=\{0\} .
$$

Thus, we find that

$$
\phi\left(D_{0}\right) \cap T M=\{0\}, \phi\left(D_{0}\right) \cap \operatorname{ltr}(T M)=\{0\}
$$

and

$$
\phi\left(D_{0}\right) \cap\{\phi(S(T M)) \perp \phi(\operatorname{ltr}(T M)) \oplus \phi(R a d T M)\}=\{0\}
$$

which shows that $D_{0}$ is invariant.

\section{ST-anti-invariant lightlike submanifolds.}

Takahasi [5] shows that it suffices to consider those indefinite almost contact manifolds with spacelike structure vector field $V$. Therefore from now on, we restrict ourselves to the case of being $V$ spacelike unit vector.

A plane section $\Pi$ in $T_{x} \bar{M}$ of a Cosymplectic manifold $\bar{M}$ is called a $\phi$ section if it is spanned by a unit vector $X$ orthogonal to $V$ and $\phi X$, where $X$ is a non-null vector field on $\bar{M}$. The sectional curvature $K(\Pi)$ with respect 
to $\Pi$ determined by $X$ is called a $\phi$-sectional curvature. If $\bar{M}$ has a $\phi$-sectional curvature $c$ which does not depend on the $\phi$-section at each point, then $c$ is constant in $\bar{M}$. Then, $\bar{M}$ is called an indefinite Cosymplectic space form and is denoted by $\bar{M}(c)$. The curvature tensor of $\bar{R}$ of $\bar{M}(c)$ for spacelike vector field $V$ is given by $[1,4]$

$$
\begin{aligned}
& \bar{R}(X, Y) Z=\frac{c}{4}\{\bar{g}(Y, Z) X-\bar{g}(X, Z) Y+\eta(X) \eta(Z) Y-\eta(Y) \eta(Z) X \\
& \quad+\bar{g}(X, Z) \eta(Y) V-\bar{g}(Y, Z) \eta(X) V+\bar{g}(\phi Y, Z) \phi X+\bar{g}(\phi Z, X) \phi Y \\
& \quad-2 \bar{g}(\phi X, Y) \phi Z)\}
\end{aligned}
$$

for any $X, Y, Z$ vector fields on $\bar{M}$.

The $\left(R_{q}^{2 m+1}, \phi_{0}, V, \eta, g\right)$ will denote the manifold $R_{q}^{2 m+1}$ with its usual Cosymplectic structure given by

$$
\left\{\begin{array}{l}
\eta=d z, \quad V=\partial z \\
\bar{g}=\eta \otimes \eta-\sum_{i=1}^{q / 2}\left(d x^{i} \otimes d x^{i}+d y^{i} \otimes d y^{i}\right)+\sum_{i=q+1}^{m}\left(d x^{i} \otimes d x^{i}+d y^{i} \otimes d y^{i}\right) \\
\phi_{0}\left(\sum_{i=1}^{m}\left(X_{i} \partial x^{i}+Y_{i} \partial y^{i}\right)+Z \partial z\right)=\sum_{i=1}^{m}\left(Y_{i} \partial x^{i}-X_{i} \partial y^{i}\right)
\end{array}\right.
$$

where $\left(x^{i}, y^{i}, z\right)$ are the Cartesian coordinates.

we have

EXAMPLE 3.1. Let $\bar{M}=\left(R_{2}^{9}, \bar{g}\right)$ be a semi-Euclidean space, where $g$ is of signature $(-,+,+,+,-,+,+,+,+)$ with respect to the canonical basis

$$
\left\{\partial x_{1}, \partial x_{2}, \partial x_{3}, \partial x_{4}, \partial y_{1}, \partial y_{2}, \partial y_{3}, \partial y_{4}, \partial z\right\} \text {. }
$$

Consider a submanifold $M$ of $R_{2}^{9}$, defined by

$$
\left\{\begin{array}{c}
x_{1}=\sin u_{1} \cosh u_{2}, \quad x_{2}=\cos u_{1} \cosh u_{2}, \\
x_{3}=\sin u_{1} \sinh u_{2}, \quad x_{4}=\cos u_{1} \sinh u_{2}, \\
x_{5}=u_{1}, \quad x_{6}=0, \quad x_{7}=\cos u_{3}, \quad x_{8}=\sin u_{3} \\
z=t .
\end{array}\right.
$$

Then a local frame of $T M$ is given by

$$
\left\{\begin{array}{c}
Z_{1}=\cos u_{1} \cosh u_{2} \partial x_{1}-\sin u_{1} \cosh u_{2} \partial x_{2}+\cos u_{1} \sinh u_{2} \partial x_{3} \\
-\sin u_{1} \sinh u_{2} \partial x_{4}+\partial x_{5}, \\
Z_{2}=\sin u_{1} \sinh u_{2} \partial x_{1}+\cos u_{1} \sinh u_{2} \partial x_{2}+\sin u_{1} \cosh u_{2} \partial x_{3}+\cos u_{1} \cosh u_{2} \partial x_{4}, \\
Z_{3}=-\sin u_{3} \partial x_{7}+\cos u_{3} \partial x_{8}, \quad Z_{4}=V=\partial z
\end{array}\right.
$$


Thus, $M$ is a 1-lightlike submanifold with $\operatorname{RadTM}=\operatorname{span}\left\{Z_{1}\right\}$, and screen distribution $S(T M)=\left\{Z_{3}, Z_{4}\right\}$. It is easy to see that $S(T M)$ is not invariant with respect to $\phi$. Since $\left\{\phi Z_{2}, \phi Z_{3}\right\}$ is non-degenerate it follows that $\phi(S(T M)) \subset S\left(T M^{\perp}\right)$.

The lightlike transversal bundle $\operatorname{ltr}(T M)$ is spanned by $N=\frac{1}{2}\left(-\cos u_{1} \cos u_{2} \partial x_{1}+\sin u_{1} \cosh u_{2} \partial x_{2}-\cos u_{1} \sinh u_{2} \partial x_{3}\right.$ $\left.+\sin u_{1} \sinh u_{2} \partial x_{4}+\partial x_{5}\right)$

and the screen transversal bundle is

$$
S\left(T M^{\perp}\right)=\operatorname{span}\left\{W_{1}=\phi N, W_{2}=\phi Z_{1}, W_{3}=\phi Z_{3}, W_{4}=\phi Z_{2}\right\}
$$

where

$$
\left\{\begin{array}{c}
W_{1}=\frac{1}{2}\left(-\sin u_{1} \cosh u_{2} \partial x_{1}-\cos u_{1} \cosh u_{2} \partial x_{2}-\sin u_{1} \sinh u_{2} \partial x_{3}\right. \\
W_{2}=\sin u_{1} \cosh u_{2} \partial x_{1}+\cos u_{1} \cosh u_{2} \partial x_{2}+\sin u_{1} \sinh u_{2} \partial x_{3} \\
\quad+\cos u_{1} \sinh u_{2} \partial x_{4}+\partial x_{6}, \\
W_{3}=-\cos u_{3} \partial x_{7}-\sin u_{3} \partial x_{8}, \\
W_{4}=-\cos u_{1} \sinh u_{2} \partial x_{1}+\sin u_{1} \sinh u_{2} \partial x_{2}-\cos u_{1} \cosh u_{2} \partial x_{3} \\
+\sin u_{1} \cosh u_{2} \partial x_{4} .
\end{array}\right.
$$

Then it is easy to see that $M$ is a $S T$-anti-invariant lightlike submanifold.

Now, we give a characterization for $S T$-anti-invariant lightlike submanifolds of indefinite Cosymplectic space forms.

THeorem 3.1. Let $M$ be a lightlike submanifold of an indefinite Cosymplectic space form $\bar{M}(c)$. Suppose that $c \neq 0$ and $\phi(\operatorname{RadTM}) \subset S\left(T M^{\perp}\right)$. Then $M$ is ST-anti-invariant lightlike submanifold if and only if

$$
\bar{g}(\bar{R}(X, Y) \xi, \phi N)=0
$$

for $X, Y \in \Gamma(S(T M)), \xi \in \Gamma(\operatorname{RadTM})$ and $N \in \Gamma(\operatorname{ltr}(T M))$.

Proof. Since $\phi(\operatorname{RadTM}) \subset S\left(T M^{\perp}\right)$, from Lemma 2.1, we have $\phi(\operatorname{ltr}(T M)) \subset S\left(T M^{\perp}\right)$. From (1.1), we have

$$
\bar{g}(\phi X, N)=-\bar{g}(X, \phi N)=0,
$$

for $X \in \Gamma(S(T M))$ and $N \in \Gamma(\operatorname{tr}(T M))$. Hence $\phi(S(T M)) \cap \operatorname{RadTM}=\{0\}$. Moreover, we find that

$$
\bar{g}(\phi X, \phi \xi)=0 \quad \text { and } \quad \bar{g}(\phi X, \phi N)=0
$$


for $X \in \Gamma(S(T M))$ and $N \in \Gamma(\operatorname{ltr}(T M))$. Hence, we get

$$
\phi(S(T M)) \cap R a d T M=\{0\} \quad \text { and } \quad \phi(S(T M)) \cap \phi(l \operatorname{tr}(T M))=\{0\} .
$$

Similarly, we can obtain that $\phi(S(T M)) \cap \operatorname{ltr}(T M)=\{0\}$.

On the other hand, since $\phi \xi \in \Gamma\left(S\left(T M^{\perp}\right)\right)$, from (3.1), we get

$$
\bar{g}(\bar{R}(X, Y) \xi, \phi N)=\frac{c}{2} \bar{g}(X, \phi Y) \bar{g}(\xi, N) .
$$

Since $c \neq 0$ and $\bar{g}(\xi, N) \neq 0$, for $\xi \in \Gamma(\operatorname{RadTM}), N \in \Gamma(\operatorname{ltr}(T M))$. Thus, $\bar{g}(\bar{R}(X, Y) \xi, \phi N)=0$ if and only if $\phi(S(T M)) \perp S(T M)$.

Therfore, $\phi(S(T M)) \subset S\left(T M^{\perp}\right)$ as $\phi(S(T M)) \cap \operatorname{ltr}(T M)=\{0\}$. Thus, the proof is complete.

If $F_{1}, F_{2}, F_{3}$ and $F_{4}$ be the projection morphisms on $\phi(R a d T M)$, $\phi(S(T M)), \phi(\operatorname{ltr}(T M))$ and $D_{0}$ respectively. Then, in view of (2.1), for $W \in \Gamma\left(S\left(T M^{\perp}\right)\right)$, we have

$$
W=F_{1} W+F_{2} W+F_{3} W+F_{4} W .
$$

On the other hand, for $W \in \Gamma\left(S\left(T M^{\perp}\right)\right)$ we write

$$
\phi W=B W+C W
$$

where $B W$ and $C W$ are tangential and transversal parts of $\phi W$. Then applying $\phi$ to (3.2), we get

$$
\phi W=\phi F_{1} W+\phi F_{2} W+\phi F_{3} W+\phi F_{4} W .
$$

Separating tangential and transversal parts in (3.4), we find

$$
B W=\phi F_{1} W+\phi F_{2} W, \quad C V=\phi F_{3} W+\phi F_{4} W .
$$

We put $\phi F_{1}=B_{1}, \phi F_{2}=B_{2}, \phi F_{3}=C_{1}$ and $\phi F_{4}=C_{2}$, we can write (3.4) as follows:

$$
\phi W=B_{1} W+B_{2} W+C_{1} W+C_{2} W,
$$

where $\quad B_{1} V \in \Gamma(\operatorname{RadTM}), B_{2} V \in \Gamma(S(T M)), C_{1} V \in \Gamma($ ltrTM $) \quad$ and $C_{2} V \in \Gamma\left(D_{0}\right)$.

THEOREm 3.2. Let M be a ST-anti-invariant lightlike submanifold of an indefinite Cosymplectic manifold $\bar{M}$. Then the induced connection is a metric connection if and only if $\nabla_{X}^{s} \phi \xi$ has no components in $\phi(S(T M))$ for $X \in \Gamma(T M)$ and $\xi \in \Gamma(R a d T M)$. 
Proof. From (1.2), we have

$$
\begin{array}{ll} 
& \bar{\nabla}_{X} \phi \xi=\left(\bar{\nabla}_{X} \phi\right) \xi+\phi\left(\bar{\nabla}_{X} \xi\right) \\
\Rightarrow & \bar{\nabla}_{X} \phi \xi=\phi\left(\bar{\nabla}_{X} \xi\right) \\
\Rightarrow & \bar{\nabla}_{X} \xi=-\phi \bar{\nabla}_{X} \phi \xi
\end{array}
$$

for $X \in \Gamma(T M)$ and $\xi \in \Gamma(\operatorname{RadTM})$.

Using (1.7), (1.9) and (3.7), we get

$$
\begin{aligned}
\nabla_{X} \xi+h^{l}(X, \xi)+h^{s}(X, \xi)=\phi A_{\phi \xi} X- & B_{1} \nabla_{X}^{s} \phi \xi-B_{2} \nabla_{X}^{s} \phi \xi \\
& -C_{1} \nabla_{X}^{s} \phi \xi-C_{2} \nabla_{X}^{s} \phi \xi-\phi D^{l}(X, \phi \xi) .
\end{aligned}
$$

Taking the tangential parts of above equation, we get

$$
\nabla_{X} \xi=-B_{1} \nabla_{X}^{s} \phi \xi-B_{2} \nabla_{X}^{s} \phi \xi .
$$

Thus assertion follows from (cf. [2], Theorem 2.4, p. 161).

\section{Radical $S T$ lightlike submanifolds.}

THEOREM 4.1. Let $M$ be a radical ST-lightlike submanifold of an indefinite Cosymplectic manifold $\bar{M}$. Then

(a) screen distribution $S(T M)$ is integrable if and only if

$$
\bar{g}\left(h^{s}(X, \phi Y), \phi N\right)=\bar{g}\left(h^{s}(\phi Y, X), \phi N\right)
$$

for $X, Y \in \Gamma(S(T M))$ and $N \in \Gamma(\operatorname{ltr}(T M))$.

(b) radical distribution is integrable if and only if

$$
\bar{g}\left(h^{s}\left(\xi_{1}, \phi X\right), \phi \xi_{2}\right)=\bar{g}\left(h^{s}\left(\xi_{2}, \phi X\right), \phi \xi_{1}\right)
$$

for $X \in \Gamma(S(T M))$ and $\xi_{1}, \xi_{2} \in \Gamma(R a d T M)$.

Proof. (a) From (1.1) and (1.2), we have $\bar{g}([X, Y], N)=\bar{g}\left(\bar{\nabla}_{X} \phi Y, \phi N\right)$ $-\bar{g}\left(\bar{\nabla}_{Y} \phi X, \phi N\right)$ for $X, Y \in \Gamma(S(T M))$ and $N \in \Gamma(\operatorname{ltr}(T M))$. Then, using (1.5) and (1.7), we get (4.1).

(b) From (1.1), (1.2), (1.13) and (1.16), we have

$$
\bar{g}\left(\left[\xi_{1}, \xi_{2}\right], X\right)=\bar{g}\left(\bar{\nabla}_{\xi_{1}} \phi \xi_{2}, \phi X\right)-\bar{g}\left(\bar{\nabla}_{\xi_{2}} \phi \xi_{1}, \phi X\right) .
$$

Then, using (1.5), we get $g\left(\left[\xi_{1}, \xi_{2}\right], X\right)=-g\left(A_{\phi \xi_{2}} \xi_{1}, \phi X\right)+g\left(A_{\phi \xi_{1}} \xi_{2}, \phi X\right)$. 
Thus, from (1.10), we obtain

$$
g\left(\left[\xi_{1}, \xi_{2}\right], \phi X\right)=\bar{g}\left(h^{s}\left(\xi_{1}, \phi X\right), \phi \xi_{2}\right)-\bar{g}\left(h^{s}\left(\xi_{2}, \phi X\right), \phi \xi_{1}\right)
$$

which proves the assertion.

THEOREM 4.2. Let $M$ be a radical ST-lightlike submanifold of an indefinite Cosymplectic manifold $\bar{M}$. Then

(a) $S(T M)$ defines a totally geodesic foliation on $M$ if and only if $g\left(h^{s}(X, \phi Y)\right)$ has no components in $\phi(R a d T M)$ for $X, Y \in \Gamma(S(T M))$.

(b) RadTM defines a totally geodesic foliation on $M$ if and only if $h^{s}\left(\xi_{1}, \phi X\right)$ has no components in $\phi(\operatorname{ltr}(T M))$ for $\xi_{1} \in \Gamma(\operatorname{RadTM})$ and $X \in \Gamma(S(T M))$.

Proof. (a) Using (1.1), (1.2), (1.5), (1.7) and (1.10), we obtain

$$
\bar{g}\left(\nabla_{X} Y, N\right)=\bar{g}\left(h^{s}(X, \phi Y), \phi N\right)
$$

which proves the assertion.

Similarly, we have (b).

Now, we have:

THEOREM 4.3. Let $M$ be a radical ST-lightlike submanifold of an indefinite Cosymplectic manifold $\bar{M}$. Then, the induced connection is a metric connection if and only if $h^{s}(X, \phi Y)$ has no components in $\phi(\operatorname{ltr}(T M))$ for $X, Y \in \Gamma(S(T M))$.

Proof. From (1.2), we have $\bar{\nabla}_{X} \xi=-\phi \bar{\nabla}_{X} \phi \xi$ for $X \in \Gamma(T M)$ and $\xi \in \Gamma(\operatorname{RadTM})$. Hence, using (1.7) and (1.9), we get

$$
\nabla_{X} \xi+h^{l}(X, \xi)+h^{s}(X, \xi)=\phi A_{\phi \xi} X-\phi \nabla_{X}^{s} \phi \xi-\phi D^{l}(X, \phi \xi) .
$$

Taking inner product in above with $Y \in \Gamma(S(T M))$, we obtain

$$
g\left(\nabla_{X} \xi, Y\right)=-g\left(A_{\phi \xi} X, \phi Y\right) .
$$

Hence, using (1.10), we get

$$
g\left(\nabla_{X} \xi, Y\right)=-\bar{g}\left(h^{s}(X, \phi Y), \phi \xi\right) .
$$

Thus, the proof is complete.

Acknowledgments. This research is partly supported by the UNIVERSITY GRANTS COMMISSION (UGC), India under a Major Research Project No. SR. 36-321/2008. The first author would like to thank the UGC for providing the financial support to pursue this research work. 


\section{REFERENCES}

[1] D. E. BLAIR, Riemannian Geometry of Contact and Symplectic Manifolds, Birkhauser, 2002.

[2] K. L. Duggal - A. Bejancu, Lightlike Submanifolds of Semi-Riemannian Manifolds and Applications, vol. 364 of Mathematics and Its Applications, Kluwer Academic Publishers, Dordrecht, The Netherlands, 1996.

[3] B. SAHIN, Screen transversal lightlike submanifolds of Indefinite Kaehler Manifolds, Chaos, Solitons and Fractals, 38 (2008), pp. 1439-1448.

[4] T. H. KANG - S. K. KIM, Lightlike hypersurfaces of indefinite Cosymplectic manifolds, International Mathematical Forum, 2, no. 67 (2007), pp. 3303-3316.

[5] T. TAKAHASHI, Sasakian manifolds with pseudo-Riemannian metric, Tohoku Math. Journal, 21 (1969), pp. 271-290.

Manoscritto pervenuto in redazione il 14 novembre 2009. 